
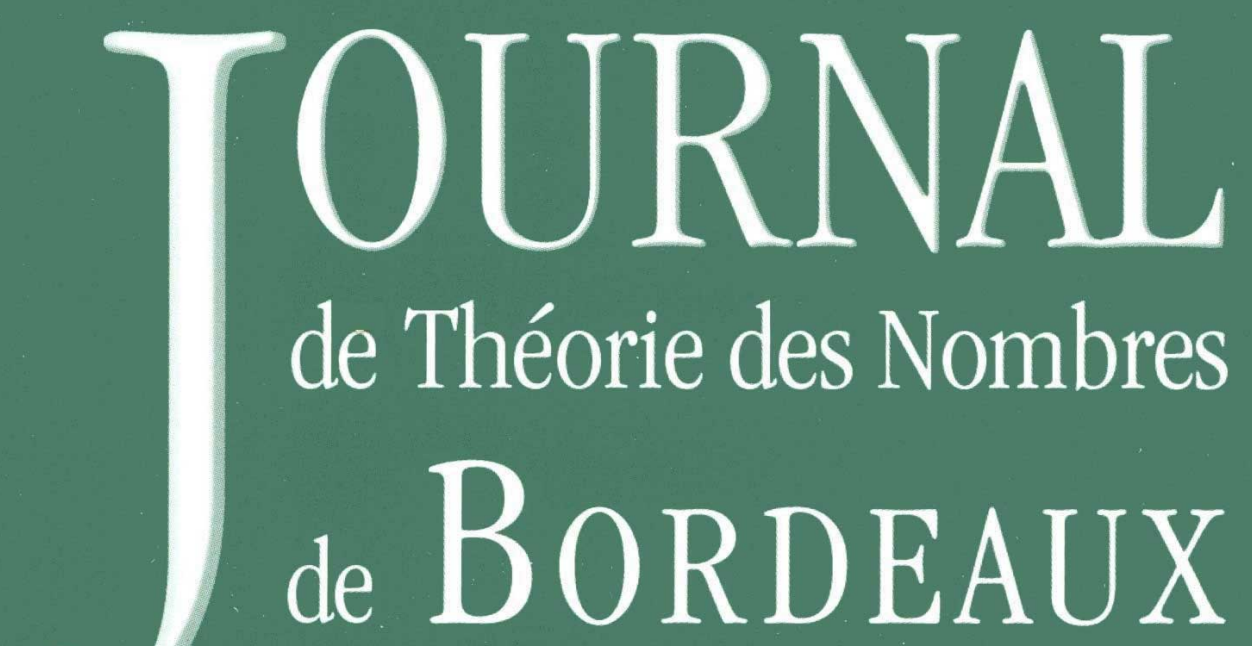

anciennement Séminaire de Théorie des Nombres de Bordeaux

Noam D. ELKIES et Scott Duke KOMINERS

\title{
Configurations of Extremal Type II Codes via Harmonic Weight
} Enumerators

Tome 31, no 3 (2019), p. 679-688.

<http://jtnb.centre-mersenne.org/item?id=JTNB_2019__31_3_679_0>

(C) Société Arithmétique de Bordeaux, 2019, tous droits réservés.

L'accès aux articles de la revue «Journal de Théorie des Nombres de Bordeaux » (http://jtnb.centre-mersenne.org/), implique l'accord avec les conditions générales d'utilisation (http://jtnb. centre-mersenne.org/legal/). Toute reproduction en tout ou partie de cet article sous quelque forme que ce soit pour tout usage autre que l'utilisation à fin strictement personnelle du copiste est constitutive d'une infraction pénale. Toute copie ou impression de ce fichier doit contenir la présente mention de copyright.

\section{cedram}

Article mis en ligne dans le cadre du

Centre de diffusion des revues académiques de mathématiques

http://www.centre-mersenne.org/ 


\title{
Configurations of Extremal Type II Codes via Harmonic Weight Enumerators
}

\author{
par NoAm D. ELKIES et ScotT Duke KOMINERS
}

RÉSUMÉ. Nous démontrons des résultats de configuration pour les codes extrêmes de Type II analogues à ceux obtenus par Ozeki et par Kominers pour les réseaux extrêmes de Type II. Plus précisément, nous démontrons que pour

$$
n \in\{8,24,32,48,56,72,96\}
$$

tout code extrême de Type II et de longueur $n$ est généré par ses mots de code de poids minimal. Là où Ozeki et Kominers utilisent des harmoniques sphériques et des fonctions thêta pondérées, nous utilisons des polynômes harmoniques discrets et des énumérateurs de poids harmoniques. En cours de route, nous introduisons la notion de $t \frac{1}{2}$-designs comme un analogue discret des dessins sphériques de Venkov portant le même nom.

Abstract. We prove configuration results for extremal Type II codes, analogous to the configuration results of Ozeki and of Kominers for extremal Type II lattices. Specifically, we show that for

$$
n \in\{8,24,32,48,56,72,96\}
$$

every extremal Type II code of length $n$ is generated by its codewords of minimal weight. Where Ozeki and Kominers used spherical harmonics and weighted theta functions, we use discrete harmonic polynomials and harmonic weight enumerators. Along the way we introduce " $t \frac{1}{2}$-designs" as a discrete analog of Venkov's spherical designs of the same name.

\section{Introduction}

We denote by $\mathbb{F}_{2}$ the two-element field $\mathbb{Z} / 2 \mathbb{Z}$. By a "code" we mean a binary linear code of length $n$, that is, a linear subspace of $\mathbb{F}_{2}^{n}$. For such a code $C$, and any integer $w$, we define

$$
C_{w}:=\{c \in C: \operatorname{wt}(c)=w\},
$$

where $\operatorname{wt}(c):=\left|\left\{i: c_{i}=1\right\}\right|$ is the Hamming weight. Recall that the dual code of $C$, denoted by $C^{\perp}$, is defined by

$$
C^{\perp}:=\left\{c^{\prime} \in \mathbb{F}_{2}^{n}:\left(c, c^{\prime}\right)=0 \text { for all } c \in C\right\},
$$

Manuscrit reçu le 31 décembre 2018, révisé le 14 octobre 2019, accepté le 25 octobre 2019.

2020 Mathematics Subject Classification. 94B05, 05B05, 11H71, 33C50.

Mots-clefs. Type II code, extremal code, $t$-design, discrete harmonic polynomial.

This work includes a part of the second author's undergraduate thesis [14]. 
where $(\cdot, \cdot)$ is the usual bilinear pairing $(x, y)=\sum_{i=1}^{n} x_{i} y_{i}$ on $\mathbb{F}_{2}^{n}$. Then $C^{\perp}$ is also linear, with $\operatorname{dim}(C)+\operatorname{dim}\left(C^{\perp}\right)=n$. A code $C$ is said to be selfdual if $C=C^{\perp}$. Such a code must have $\operatorname{dim}(C)=n / 2$; in particular $2 \mid n$. Because $(c, c) \equiv \operatorname{wt}(c) \bmod 2$, it follows that a self-dual code $C$ is even, that is, $C$ has $2 \mid \operatorname{wt}(c)$ for every $c \in C$; equivalently, $C_{w}=\emptyset$ unless $2 \mid w$. A self-dual code $C$ is said to be doubly even, or of Type II, if $4 \mid \operatorname{wt}(c)$ for all $c \in C$; equivalently, if $C_{w}=\emptyset$ unless $4 \mid w$. It is well-known that the length of a Type II code is always a multiple of 8 .

Mallows and Sloane [16] showed that a Type II code $C$ of length $n$ must contain nonzero codewords of weight at most $4\lfloor n / 24\rfloor+4$ (see also [5, p. 194]). If a Type II code $C$ of length $n$ has $C_{w}=\emptyset$ for all positive $w<4\lfloor n / 24\rfloor+4$, then $C$ is said to be extremal, because such a code has the largest minimal weight among all Type II codes of its length.

In this paper, we prove configuration results for extremal Type II codes. Specifically, we show that if $C$ is an extremal Type II code of length $n=8,24,32,48,56,72$, or 96 , then $C$ is generated by its minimal-weight codewords. Our approach uses the machinery of harmonic weight enumerators introduced by Bachoc [2] and developed further in [7], following the approach used to prove analogous results for lattices in the works of Venkov [26], Ozeki [18, 19], and the second author [13].

For each $n$ that we consider, it is known that the words of each weight in an extremal code of length $n$ form a combinatorial $\mathrm{t}(n)$-design (with $\mathrm{t}(n)$ as defined in (2.3)), just as the vectors of each norm in an extremal Type II lattice of length $n$ form a spherical $(2 \mathrm{t}(n)+1)$-design. For some of the $n$ that we consider $(n=56$ and 96$)$, the $(2 \mathrm{t}(n)+1)$-design property was not enough to prove the configuration result for lattices. Luckily, the vectors of a given norm are known to satisfy a further constraint, called the " $\left(2 \mathrm{t}(n)+1 \frac{1}{2}\right)$-design" condition by Venkov [27], which made it possible to extend the configuration result to $n=56$ and $n=96$ as well. Our proofs for codes use an analogous further constraint (see Definition 2.6) to handle the cases $n=56$ and 96 .

After we first circulated our results in [14] and [7], we learned that for each $n$ we consider, our configuration result had already been obtained [21, 25, 12, 9, 11, 8] using Mendelsohn's relations [17] — that is, using only the fact that the words of each weight in an extremal code of length $n$ form a combinatorial $\mathrm{t}(n)$-design. ${ }^{1}$ Mendelsohn's relations give a system of linear equations that forces some counts to be fractional (where in the setting of lattices of ranks 56 and 96 the counts were all integral, so we could not reach a contradiction without using the $\left(2 \mathrm{t}(n)+1 \frac{1}{2}\right)$-design constraint). We believe that the new proofs we present here are still of interest because

\footnotetext{
${ }^{1}$ See also [10], which uses Mendelsohn's relations to prove a further generalization of some of these configuration results.
} 
they further the analogy between codes and lattices of Type II by using harmonic weight enumerators and the $t \frac{1}{2}$-design property.

\section{Designs, Extremal Codes, and Discrete Harmonic Polynomials}

Fix a positive integer $n$. For each nonnegative integer $w \leq n$, denote by $\Omega_{w}$ the Hamming sphere of radius $w$ about the origin of $\mathbb{F}_{2}^{n}$. Thus $\Omega_{w}$ consists of the $\left(\begin{array}{l}n \\ w\end{array}\right)$ binary words of length $n$ and weight $w$. To such a word $c$ we associate its support $\Sigma_{c}:=\left\{i: 1 \leq i \leq n, c_{i}=1\right\}$, a $w$-element set.

We use the following definition of a $t$-design in $\Omega_{w}$, which assumes neither that $w \geq t$ nor that the design is nonempty.

Definition 2.1. We say that a subset $D \subseteq \Omega_{w}$ is a t-predesign for an integer $t \geq 0$ if there exists an integer $N=N_{t}(D)$ such that every subset $I \subset\{1,2, \ldots, n\}$ of cardinality $t$ is contained in exactly $N$ of the sets $\Sigma_{c}$ with $c \in D$. Then a subset $D \subseteq \Omega_{w}$ is called a $t$-design if $D$ is a $t^{\prime}$-predesign for each positive integer $t^{\prime} \leq t$.

Remarks. It is well known that if $w \geq t$, then $D \subseteq \Omega_{w}$ is a $t$-design if and only if

$$
\left(\begin{array}{l}
n \\
w
\end{array}\right) \sum_{c \in D} f(c)=|D| \sum_{c \in \Omega_{w}} f(c)
$$

for any function $f: \mathbb{F}_{2}^{n} \rightarrow \mathbb{C}$ that depends on at most $t$ of the $n$ coordinates, and that $N_{t}(D)$ is given by the formula

$$
\left(\begin{array}{l}
n \\
t
\end{array}\right) N_{t}(D)=\left(\begin{array}{l}
w \\
t
\end{array}\right)|D|
$$

because both sides of $(2.2)$ count ordered pairs $(I, c)$ such that $|I|=t$, $c \in D$, and $I \subseteq \Sigma_{c}$. In this case a $t$-predesign $D$ is automatically a $t$-design, but if $w<t$ then every subset of $\Omega_{w}$ is a $t$-predesign (with $N_{t}(D)=0$, still in accordance with (2.2)), so we need the "predesign" property also for $t^{\prime}<t$ to assure that a $t$-design is also a $t^{\prime}$-design for $t^{\prime}<t$. Moreover, the only $w$-predesigns in $\Omega_{w}$ are $\Omega_{w}$ itself and $\emptyset$, so once $t \geq w$ it follows that the only $t$-designs in $\Omega_{w}$ are $\Omega_{w}$ itself and $\emptyset$. It follows that (2.1) still holds for $t \geq w$.

Extremal codes yield designs by the following important special case of the Assmus-Mattson theorem. For $n \equiv 0 \bmod 8$, we define $\mathrm{t}(n)$ by

$$
\mathrm{t}(n):= \begin{cases}5 & n \equiv 0 \bmod 24 \\ 3 & n \equiv 8 \bmod 24 \\ 1 & n \equiv 16 \bmod 24\end{cases}
$$


Theorem 2.2 ([1]). If $C$ is an extremal Type II code of length $n$, then $C_{w}$ is a $\mathrm{t}(n)$-design for each $w$.

In [7, Thm. 7.4], we gave a new proof of Theorem 2.2 using the discrete harmonic polynomials $Q: \mathbb{F}_{2}^{n} \rightarrow \mathbb{C}$ introduced by Delsarte [6], via his characterization of $t$-designs:

Theorem 2.3 ([6, Thm. 7], [7, Prop. 7.1]). A set $D \subseteq \Omega_{w}$ is a t-design if and only if

$$
\sum_{v \in D} Q(v)=0
$$

for all nonconstant discrete harmonic polynomials $Q$ with $\operatorname{deg} Q \leq t$.

We note two important corollaries of Theorem 2.3. The first reorganizes (2.4):

Corollary 2.4 ([6, Thm. 6], [7, Cor. 7.3]). A set $D \subseteq \Omega_{w}$ is a t-design if and only if

$$
\sum_{v \in D} Q(v)=\frac{|D|}{\left|\Omega_{w}\right|} \sum_{v \in \Omega_{w}} Q(v)
$$

for all discrete harmonic polynomials $Q$ with $\operatorname{deg} Q \leq t$.

Note that $\sum_{v \in \Omega_{w}} Q(v)$, and thus also $\sum_{v \in D} Q(v)$, vanishes unless $\operatorname{deg} Q=0$.

The second corollary is the special case of (2.4) when $Q$ is a discrete zonal harmonic polynomial, that is, a discrete harmonic polynomial such that $Q(v)$ depends only on the weights of $v$ and $v \cap \dot{v}$ for some fixed vector $\dot{v}$ (equivalently, $Q(v)$ depends only on $\operatorname{wt}(v)$ and the distance between $v$ and $\dot{v}$ ). Given a degree $d$ and a fixed $\dot{v} \in \mathbb{F}_{2}^{n}$, we showed in [7, Sec. 6] that there is a one-dimensional space of discrete zonal harmonic polynomials, generated by

$$
Q_{d ; \dot{v}}(v):=\sum_{k=0}^{d}(-1)^{k}\left(\prod_{\ell=0}^{k-1} \frac{(n-\mathrm{wt}(\dot{v}))-(d-\ell-1)}{\operatorname{wt}(\dot{v})-\ell}\right) Q_{d, k ; \dot{v}}(v),
$$

where

$$
\begin{aligned}
Q_{d, k ; \dot{v}}(v) & =\left(\sum_{i=0}^{k}(-1)^{i}\left(\begin{array}{c}
\mathrm{wt}(v \cap \dot{v}) \\
i
\end{array}\right)\left(\begin{array}{c}
\mathrm{wt}(\dot{v})-\mathrm{wt}(v \cap \dot{v}) \\
k-i
\end{array}\right)\right) \\
\times & \left(\sum_{i=0}^{d-k}\left(\begin{array}{c}
\operatorname{wt}(v)-\mathrm{wt}(v \cap \dot{v}) \\
i
\end{array}\right)\left(\begin{array}{c}
(n-\mathrm{wt}(\dot{v}))-(\operatorname{wt}(v)-\mathrm{wt}(v \cap \dot{v})) \\
d-k-i
\end{array}\right)\right) .
\end{aligned}
$$

Corollary 2.5 ([7, Cor. 7.6]). If $D \subseteq \Omega_{w}$ is a t-design then

$$
\sum_{v \in D} Q_{d ; \dot{v}}(v)=0
$$


for each positive $d \leq t$ and any $\dot{v} \in \mathbb{F}_{2}^{n}$.

The approach to Theorem 2.2 via discrete harmonic polynomials is motivated by the fruitful analogy between Type II codes and Type II lattices, which are even unimodular Euclidean lattices. Recall [23, Ch. VII] that the rank of a Type II lattice $L$ must be a multiple of 8 , and that its theta function is a modular form for $\mathrm{PSL}_{2}(\mathbb{Z})$. It follows via a theorem of Siegel [24] that if $L$ has rank $n$ then its minimal nonzero norm is at most $2\lfloor n / 24\rfloor+2$ (Mallows-Odlyzko-Sloane [15]). If equality holds then $L$ is said to be $e x$ tremal. In such a lattice the vectors of each given norm form a spherical $(2 \mathrm{t}(n)+1)$-design. As in Corollary 2.4, this means that the sum over those vectors of $P$ vanishes for any nonconstant harmonic polynomial $P$ of degree at most $2 \mathrm{t}(n)+1$. The $(2 \mathrm{t}(n)+1)$-design property is proved by recognizing the sum as a coefficient of a modular form (a weighted theta function); our proof of Theorem 2.2 in [7] is analogous, using harmonic weight enumerators of Type II codes.

In the lattice setting, the modular-form approach gives additional information on the configuration of lattice vectors of given norm, beyond the fact that the configuration is a $(2 \mathrm{t}(n)+1)$-design. Namely, while the sum of a spherical harmonic of degree $2 \mathrm{t}(n)+2$ over lattice vectors of a given norm need not vanish (i.e., those vectors need not constitute a $(2 \mathrm{t}(n)+2)$-design), a spherical harmonic of degree $2 \mathrm{t}(n)+4$ does sum to 0 . (Odd harmonics sum to 0 automatically because the design is centrally symmetric.) Venkov [27] calls such a spherical configuration a " $\left(2 \mathrm{t}(n)+1 \frac{1}{2}\right)$-design". In [7, Prop. 7.5], we proved that for an extremal Type II code $C$ each $C_{w}$ satisfies an additional constraint, analogous to the $\left(2 \mathrm{t}(n)+1 \frac{1}{2}\right)$-design property of extremal lattices. We thus introduce parallel terminology in this setting. Recall (Theorem 2.3) that $D \subseteq \Omega_{w}$ is a $t$-design if and only if $\sum_{v \in D} Q(v)=0$ for all nonconstant discrete harmonic polynomials $Q$ of degree at most $t$.

Definition 2.6. A subset $D \subseteq \Omega_{w}$ is said to be a $t \frac{1}{2}$-design if $D$ is a $t$-design such that in addition $\sum_{v \in D} Q(v)=0$ holds for all discrete harmonic polynomials $Q$ of degree $t+2$.

Then the result from [7] can be expressed as follows:

Theorem 2.7 ([7, Prop. 7.5]). Let $\mathrm{t}=\mathrm{t}(n)$. If $C$ is an extremal Type II code of length $n$, then $C_{w}$ is a $\frac{1}{2}$-design for each $w$. In particular, for each $w$ and any $\dot{v} \in \mathbb{F}_{2}^{n}$,

$$
\sum_{v \in C_{w}} Q_{d ; \dot{v}}(v)=0
$$

holds for positive $d \leq \mathrm{t}$ and also for $d=\mathrm{t}+2$. 


\section{Configuration Results}

3.1. Preliminaries. Throughout this section, $C$ denotes a length- $n$ extremal Type II code, and $w_{0}:=\min (C)$ denotes the minimal weight of nonzero codewords in $C$.

We denote by $\mathcal{C}_{w}(C)$ the linear subcode of $C$ generated by $C_{w}$. For any $\dot{v} \in \mathbb{F}_{2}^{n}$ and any $j(0 \leq j \leq n)$, we denote by $N_{j}(C ; \dot{v})$ the value

$$
N_{j}(C ; \dot{v}):=\left|\left\{c \in \mathcal{C}_{w_{0}}(C): \operatorname{wt}(c \cap \dot{v})=j\right\}\right| .
$$

For $c \in C^{\perp}$, we must have $N_{2 j^{\prime}+1}(C ; c)=0$ for all $j^{\prime}$.

Lemma 3.1. If $\dot{c}$ is a minimal-weight representative of the class $[\dot{c}] \in$ $C / \mathcal{C}_{w_{0}}(C)$ and $c \in C_{w_{0}}$, then

$$
\mathrm{wt}(c \cap \dot{c}) \leq \frac{w_{0}}{2} .
$$

Proof. If $\mathrm{wt}(c \cap \dot{c})>w_{0} / 2$, then $[\dot{c}]$ contains a codeword $c+\dot{c}$ of weight

$$
\mathrm{wt}(c+\dot{c})=\mathrm{wt}(c)+\mathrm{wt}(\dot{c})-2 \mathrm{wt}(c \cap \dot{c})<\mathrm{wt}(\dot{c}) ;
$$

this contradicts the minimality of $\dot{c}$ in $[\dot{c}]$.

3.2. Extremal Type II Codes of Lengths 48 and 72. We begin with a configuration result for Type II codes of lengths $n=48$ and 72 .

Theorem 3.2. If $C$ is an extremal Type II code of length $n=48$ or 72 , then

$$
C=\mathcal{C}_{w_{0}}(C) .
$$

Proof. We consider the equivalence classes of $C / \mathcal{C}_{w_{0}}(C)$ and assume for the sake of contradiction that there is some class $[\dot{c}] \in C / \mathcal{C}_{w_{0}}(C)$ with minimalweight representative $\dot{c}$ having $\operatorname{wt}(\dot{c})=s>w_{0}$.

As $C$ is self-dual, we have $N_{2 j^{\prime}+1}(C ; c)=0$ for all $0 \leq j^{\prime} \leq\lfloor n / 2\rfloor$. Additionally, by Lemma 3.1, we must have $N_{2 j^{\prime}}(C ; \dot{c})=0$ for $j^{\prime}>w_{0} / 4$. We now develop a system of equations in the

$$
\frac{w_{0}}{4}+1
$$

variables $N_{0}(C ; \dot{c}), N_{2}(C ; \dot{c}), \ldots, N_{w_{0} / 2}(C ; \dot{c})$.

Combining the $\mathrm{t}(n)+1$ equations of Corollary 2.5 with the equation

$$
N_{0}(C ; \dot{c})+N_{2}(C ; \dot{c})+\cdots+N_{w_{0} / 2}(C ; \dot{c})=\left|C_{w_{0}}\right|
$$

gives a system of

$$
\mathrm{t}(n)+2>\frac{w_{0}}{4}+1
$$

equations in the variables $N_{2 j^{\prime}}(C ; \dot{c})\left(0 \leq j^{\prime} \leq w_{0} / 4\right)$. 
For $n=48,72$, the (extended) determinants of these inhomogeneous systems are

$$
\begin{gathered}
2^{26} 3^{5} 5^{2} 7^{1} 11^{2} 23^{2} 43^{1} 47^{1}\left(\frac{11 s^{3}-396 s^{2}+4906 s-20736}{(s-3)(s-2)^{2}(s-1)^{3} s^{3}}\right) \\
2^{42} 3^{5} 5^{2} 7^{2} 11^{2} 13^{1} 17^{3} 23^{2} 67^{2} 71^{1} \\
\left(\frac{39 s^{4}-2600 s^{3}+67410 s^{2}-800440 s+3650496}{(s-4)(s-3)^{2}(s-2)^{3}(s-1)^{4} s^{4}}\right)
\end{gathered}
$$

respectively ${ }^{2}$ these determinants must vanish, as they are derived from overdetermined systems. Since equations (3.2)-(3.3) have no integer roots $s$, we have reached a contradiction.

3.3. Extremal Type II Codes of Length At Most 32. The approach used to prove Theorem 3.2 may also be applied to show that extremal Type II codes of lengths $n=8,24$, and 32 are generated by their minimalweight codewords. In these cases the determinants

$$
\begin{gathered}
2^{7} 3^{1} 7^{1}\left(\frac{3 s-10}{(s-1) s}\right), \\
2^{15} 3^{2} 5^{1} 7^{1} 11^{2} 23^{1}\left(\frac{7 s^{2}-98 s+344}{(s-2)(s-1)^{2} s^{2}}\right), \\
2^{17} 3^{1} 5^{2} 7^{1} 29^{1} 31^{1}\left(\frac{7 s^{2}-126 s+584}{(s-2)(s-1)^{2} s^{2}}\right)
\end{gathered}
$$

are obtained; none have integral roots $s$. We therefore recover the following result.

Theorem 3.3. If $C$ is an extremal Type II code of length $n=8,24$, or 32, then $C=\mathcal{C}_{w_{0}}(C)$.

Technically, Theorem 3.3 has been known for a long time, as the extremal Type II codes of lengths $n=8,24$, and 32 have been fully classified [3, 4 , 20, 21, 22]. Our methods, however, let us prove that the extremal Type II codes of these lengths are generated by their minimal codewords without appeal to the classification results or to the explicit forms of those codes.

There is no analog of Theorems 3.2 and 3.3 for extremal Type II codes of length $n=16$. Indeed, the extremal Type II code with tetrad subcode $d_{16}$ has codewords of weight 8 that cannot be obtained as linear combinations

\footnotetext{
${ }^{2}$ These determinants were computed using the formula of Corollary 2.5. We omit the equations obtained from the zonal spherical harmonic polynomials of the highest degrees when there are more than $\frac{w_{0}}{4}+2$ equations obtained by this method.
} 
of codewords of weight 4 . As expected, following the method used to prove Theorem 3.2 in the case $n=16$ yields the determinant

$$
-93184\left(\frac{s-8}{(s-1) s}\right)
$$

which vanishes for $s=8$.

3.4. Extremal Type II Codes of Lengths 56 and 96. Now, we prove an analog of Theorems 3.2 and 3.3 for extremal Type II codes of lengths $n=56$ and 96 .

Theorem 3.4. If $C$ is an extremal Type II code of length $n=56$ or 96, then

$$
C=\mathcal{C}_{w_{0}}(C)
$$

Proof. Seeking a contradiction, we suppose that $\mathcal{C}_{w_{0}}(C) \subsetneq C$. Then

$$
\mathcal{C}_{w_{0}}(C)^{\perp} \supsetneq C^{\perp}=C \supsetneq \mathcal{C}_{w_{0}}(C) .
$$

Thus, there is some equivalence class $[\dot{c}] \in \mathcal{C}_{w_{0}}(C)^{\perp} / \mathcal{C}_{w_{0}}(C)$ with minimalweight representative $\dot{c}$ of weight $\operatorname{wt}(\dot{c})=s>0$.

Corollary 2.5 yields $\mathrm{t}(n)+1$ equations in the variables $N_{2 j^{\prime}}(C ; \dot{c})(0 \leq$ $\left.j^{\prime} \leq w_{0} / 4\right) .{ }^{3}$ Combining these equations with (3.1), we obtain a system of $\mathrm{t}(n)+2$ equations in the

$$
\frac{w_{0}}{4}+1<\mathrm{t}(n)+2
$$

variables $N_{2 j^{\prime}}(C ; \dot{c})\left(0 \leq j^{\prime} \leq w_{0} / 4\right)$. For $n=56,96$, these inhomogeneous systems have (extended) matrices with determinants

$$
\begin{gathered}
-2^{27} 3^{7} 5^{3} 7^{3} 11^{1} 13^{2} 17^{1} 53^{1}\left(\frac{(s-16)\left(3 s^{3}-112 s^{2}+1368 s-5120\right)}{(s-4)(s-3)(s-2)^{2}(s-1)^{3} s^{3}}\right) \\
-2^{59} 3^{9} 5^{4} 7^{2} 11^{2} 13^{2} 17^{1} 19^{1} 23^{3} 29^{1} 31^{2} 43^{1} 47^{2} 89^{2} \cdot S_{96}(s)
\end{gathered}
$$

where $S_{96}$ is the rational function

$$
S_{96}(s)=\frac{(s-24)\left(68 s^{5}-6936 s^{4}+289901 s^{3}-6153306 s^{2}+65640728 s-277774080\right)}{(s-6)(s-5)(s-4)^{2}(s-3)^{3}(s-2)^{4}(s-1)^{5} s^{5}} .
$$

These determinants must vanish, but the only integral roots of (3.5) and (3.6) are multiples of 4 . Therefore, $\mathcal{C}_{w_{0}}(C)^{\perp}$ is doubly even, and it follows that $\mathcal{C}_{w_{0}}(C)^{\perp}=\mathcal{C}_{w_{0}}(C)$, contradicting (3.4). We must therefore have $\mathcal{C}_{w_{0}}(C)=C$.

\footnotetext{
${ }^{3}$ Note that the variables $N_{j}(C ; \dot{c})$ vanish for $j$ not of the form $2 j^{\prime}$ with $0 \leq j^{\prime} \leq w_{0} / 4$, as the conclusion of Lemma 3.1 holds for $[\dot{c}] \in \mathcal{C}_{w_{0}}(C)^{\perp} / \mathcal{C}_{w_{0}}(C)$.
} 
Acknowledgements. The authors thank Zachary Abel, Henry Cohn, John H. Conway, Alex Cowan, Ben Green, Benedict H. Gross, Barry Mazur, Gabriele Nebe, Ken Ono, Vera Pless, Eric M. Rains, and the referee for helpful comments and suggestions. During parts of this research, Elkies was supported by NSF grants DMS-0501029, DMS-1100511, and DMS-1502161, a Radcliffe Fellowship, and the Simons Collaboration on Arithmetic Geometry, Number Theory, and Computation; Kominers was supported by the Harvard College Program for Research in Science and Engineering (PRISE), a Harvard Mathematics Department Highbridge Fellowship, an NSF Graduate Research Fellowship, NSF grants CCF-1216095 and SES-1459912, an AMS-Simons Travel Grant, the Harvard Milton Fund, and the Ng Fund and the Mathematics in Economics Research Fund of the Harvard Center of Mathematical Sciences and Applications.

\section{References}

[1] E. F. Assmus \& H. F. Mattson, "New 5-designs", J. Comb. Theory 6 (1969), p. 122-151.

[2] C. BACHOC, "On harmonic weight enumerators of binary codes", Des. Codes Cryptography 18 (1999), p. 11-28.

[3] J. H. Conway \& V. Pless, "On the enumeration of self-dual codes", J. Comb. Theory, Ser. A 28 (1980), p. 26-53.

[4] - "The binary self-dual codes of length up to 32: A revised enumeration", J. Comb. Theory, Ser. A 60 (1992), p. 183-195.

[5] J. H. Conway \& N. J. A. Sloane, Sphere Packings, Lattices and Groups, 3rd ed., Springer, 1999.

[6] P. Delsarte, "Hahn polynomials, discrete harmonics, and t-designs", SIAM J. Appl. Math. 34 (1978), p. 157-166.

[7] N. D. Elkies \& S. D. Kominers, "Weighted Generating Functions for Type II Lattices and Codes", in Quadratic and Higher Degree Forms, Developments in Mathematics, vol. 31, Springer, 2013, p. 63-108.

[8] M. Harada, "Remark on a 5-design related to a putative extremal doubly-even self-dual [96, 48, 20] code", Des. Codes Cryptography 37 (2005), p. 355-358.

[9] — "Self-orthogonal 3- $(56,12,65)$ designs and extremal doubly-even self-dual codes of length 56", Des. Codes Cryptography 38 (2006), p. 5-16.

[10] - "On a 5-design related to a putative extremal doubly even self-dual code of length a multiple of 24", Des. Codes Cryptography 76 (2015), p. 373-384.

[11] M. Harada, M. Kitazume \& A. Munemasa, "On a 5-design related to an extremal doubly even self-dual code of length 72", J. Comb. Theory, Ser. A 107 (2004), p. 143-146.

[12] M. Harada, A. Munemasa \& V. D. Tonchev, "A characterization of designs related to an extremal doubly-even self-dual code of length 48", Ann. Comb. 9 (2005), p. 189-198.

[13] S. D. Kominers, "Configurations of Extremal Even Unimodular Lattices", Int. J. Number Theory 5 (2009), p. 457-464.

[14] - "Weighted Generating Functions and Configuration Results for Type II Lattices and Codes", Undergraduate Thesis, Harvard University, 2009.

[15] C. L. Mallows, A. M. Odlyzko \& N. J. A. Sloane, "Upper bounds for modular forms, lattices and codes", J. Algebra 36 (1975), p. 68-76.

[16] C. L. Mallows \& N. J. A. Sloane, "An upper bound for self-dual codes", Inform. and Control 22 (1973), p. 188-200.

[17] N. S. Mendelsohn, "Intersection numbers of $t$-designs", in Studies in Pure Mathematics: Papers in Combinatorial Theory, Analysis, Geometry, Algebra, and the Theory of Numbers presented to Richard Rado on the Occasion of his Sixty-Fifth Birthday, Academic Press Inc., 1971, p. 145-150. 
[18] M. OzEKI, "On even unimodular positive definite quadratic lattices of rank 32", Math. Z. 191 (1986), p. 283-291.

[19] , "On the configurations of even unimodular lattices of rank 48", Arch. Math. 46 (1986), p. 54-61.

[20] V. Pless, "A classification of self-orthogonal codes over GF(2)", Discrete Math. 3 (1972), p. 209-246.

[21] , Introduction to the Theory of Error-Correcting Codes, 3rd ed., John Wiley \& Sons, 1998.

[22] V. Pless \& N. J. A. Sloane, "On the Classification and Enumeration of Self-Dual Codes", J. Comb. Theory, Ser. A 18 (1975), p. 313-335.

[23] J.-P. Serre, A Course in Arithmetic, Springer, 1973.

[24] C. L. SiEgel, "Berechnung von Zetafunktionen an ganzzahligen Stellen", Nachr. Ges. Wiss. Göttingen, Math.-Phys. Kl. 1969 (1969), p. 87-102, [pages 82-97 in Gesammelte Abhandlungen IV, Berlin: Springer 1979].

[25] V. D. Tonchev, "Quasi-symmetric 2- $(31,7,7)$ designs and a revision of Hamada's conjecture", J. Comb. Theory, Ser. A 42 (1986), p. 104-110.

[26] B. B. Venkov, "Even unimodular Euclidean lattices in dimension 32", J. Math. Sci., New York 26 (1984), p. 1860-1867.

[27] —, "Réseaux et designs sphériques", in Réseaux Euclidiens, Designs Sphériques et Formes Modulaires, Monographie de L'Enseignement Mathématique, vol. 37, L'Enseignement Mathématique, 2001 (in French), p. 10-86.

\author{
Noam D. Elkies \\ Department of Mathematics \\ Harvard University \\ One Oxford Street \\ Cambridge, MA 02138, USA \\ E-mail: elkies@math.harvard.edu \\ URL: http://www.math.harvard.edu/ elkies/
}

Scott Duke Kominers

Harvard Business School, Department of Economics, and

Center of Mathematical Sciences and Applications

Harvard University

Rock Center for Entrepreneurship

Soldiers Field, Boston, MA 02163, USA

E-mail: kominers@fas .harvard.edu

$U R L:$ http://www. scottkom.com/ 